\title{
Nothing is wrong with descriptive papers
}

\author{
Werner Paulus
}

Published online: 6 June 2014

(C) Springer-Verlag Berlin Heidelberg 2014

When I read through a referee's comments and I find the word "descriptive" or even "merely descriptive" in the initial sentences of the review, I know that in the end the referee will very probably be negative and suggest that the paper be rejected. Here, "descriptive" apparently means boring, old-fashioned, static, low-quality or simply dislike, although semantically it indicates careful observation. The "good" counterpart tends to be the word "mechanistic", meaning model-based experimental studies that analyze pathogenesis, although it is acknowledged that both "descriptive" and "mechanistic" are somewhat vaguely defined terms, and there is no clear line of demarcation between the two $[1,2]$.

In fact, it has usually been the observational rather than the mechanistic study that has moved neuropathology (and science in general) ahead. Furthermore, surgical neuropathology, as well as descriptive neuropathology research using human tissues, has become more and more clinically relevant. For example, the most frequently cited and influential original papers published during the past 10 years in this journal have all been descriptive studies using human tissues, such as those on TDP-43, IDH1, and C9ORF72. In fact, the Acta paper with the highest impact ever with more than 5,400 citations so far is a classical descriptive paper based on autopsy brains, namely the Braak and Braak staging of Alzheimer's disease published in 1991.

Studies analyzing mechanisms of disease can tell a good story and may be more interesting, while a descriptive study provides the facts. The other way round, descriptive studies may be boring and tend to overinterpret findings, while the mechanistic approach helps to understand pathogenesis and eventually could result in new therapeutic approaches. In other words, mechanistic studies tend to be more entertaining,

W. Paulus $(\bowtie)$

Institute of Neuropathology, University Hospital Münster,

Münster, Germany

e-mail: werner.paulus@uni-muenster.de while descriptive studies often tend to be more relevant. This means that mechanistic studies need to be backed up by a reality check to make them relevant, and neuropathology is in a unique position to undertake mechanistic studies AND verification using human specimens. Studies on mechanisms without checking against the reality of human tissues may be an excellent science, but may be in danger of becoming a kind of neuroscience in the clinical nirvana, and do not represent neuropathology. This self-conception is reflected in the subtitle of Acta Neuropathologica, namely "Pathology and Mechanisms of Neurological Disease". Accordingly, we love to publish excellent, interesting AND relevant papers analyzing mechanisms of disease using in vitro or in vivo models and complemented by appropriate analyses of human tissues. The same high priority is granted to excellent descriptive papers on tissue-based morphological or molecular features of neurological disease bearing an important clinical impact. Mechanistic studies without verification in human tissues may be important and can also be published in this journal, but their priority is somewhat lower if there are no apparent clinical implications. The same is true for descriptive studies using animal models without providing experimental insight into pathogenesis.

Neuropathology is not merely neuroscience of neurological disease. Neuropathology is identifying problems through the examination of human pathological material, then designing experiments to test mechanistic hypotheses and finally confirming the relevance of these hypotheses by expert re-examination of human material. Acta Neuropathologica is about to promote this view.

\section{References}

1. Casadevall A, Fang FC (2008) Descriptive science. Infect Immun 76:3835-3836

2. Casadevall A, Fang FC (2009) Mechanistic science. Infect Immun 77:3517-3519 